\title{
¿Qué es aplicar derecho?
}

\author{
What Is It to Apply the Law?*
}

Luís Duarte d'Almeida ${ }^{* *}$

Recepción: 08/03/2020

Evaluación: 14/04/2020

Aceptación final: 12/11/2020

Resumen: Este artículo ofrece una teoría de la aplicación del derecho, y un modelo de justificación de las decisiones judiciales que aplican derecho. Considera tres conjuntos de preguntas. Uno refiere al objeto directo de la aplicación del derecho: cuando un tribunal aplica el derecho, ¿qué es aquello que está aplicando exactamente? Un segundo conjunto de preguntas refiere al objeto indirecto de la aplicación del derecho: los tribunales no solo aplican derecho, sino que lo aplican $a$ algo. ¿A qué, exactamente? ¿A casos? ¿A hechos? ¿A los "hechos del caso concreto" que tienen ante sí, como suele decirse? En tercer lugar, se trata de preguntas sobre el contenido de la aplicación del derecho. ¿Es "aplicación del derecho" el nombre de un tipo específico de acto? Si es así, ¿cuáles son sus rasgos distintivos? El artículo sostiene que hay en realidad dos tipos de aplicación del derecho: la aplicación inferencial y la aplicación pragmática del derecho. La primera

Traducción de Laura Bavestrello y Cristián González. Corregida por Sebastián Figueroa y revisada por el autor. La versión en inglés de este artículo ha sido publicada en Law and Philosophy, 40(4), 361-386, 2021.

** Professor of Jurisprudence, Edinburgh School of Law, The University of Edinburgh, Reino Unido. Correo electrónico: luis.duarte.almeida@ed.ac.uk. Gracias a Antony Duff, Euan MacDonald, Gonçalo de Almeida Ribeiro, Hasan Dindjer, Lucas Miotto, Maggie O’Brien, Sebastián Figueroa, y a audiencias de Edimburgo, Florencia, Glasgow, Lisboa, Milán y Oxford, y a los revisores anónimos, por sus muy útiles comentarios sobre un borrador anterior. Comencé a pensar sobre el tema del presente trabajo cuando fui Fernand Braudel Research Fellow en el European University Institute de Florencia, en la primavera de 2017. Agradezco al EUI por haberme acogido y por las excelentes condiciones que disfruté durante mi estancia. 
se analiza en la sección 2, la segunda en la sección 3, y la relación entre ambas en la sección 4. En la sección 5 se examina la noción de "caso" y en la sección 6 se retoman los tres conjuntos de preguntas pertinentes.

Palabras clave: aplicación del derecho, aplicabilidad, justificación de decisiones judiciales, aplicación inferencial del derecho, aplicación pragmática del derecho, casos.

\begin{abstract}
This paper offers a jurisprudential account of law-application, and a model of the justification of law-applying judicial decisions. It considers three sets of questions. One concerns the direct object of law-application: when a court applies the law, what exactly is it applying? A second set of questions regards law-application's indirect object: courts do not just apply the law; they apply it to something. To what, exactly? To cases? To facts? To the "facts of the particular case" before them, as the common phrase goes? Third, questions about the content of law-application. Is "applying the law" the name of a specific type of act? If so, what are its distinctive features? There are actually - this paper argues-two kinds of law-application: inferential law-application and pragmatic law-application, as the paper calls them. The former is analysed in Section 2, the latter in Section 3, and the relation between them in Section 4. Section 5 looks into the notion of a "case", and Section 6 returns to the three relevant sets of questions.
\end{abstract}

Keywords: law-application, applicability, justification of judicial decisions, pragmatic law-application, inferential law-application, cases.

\title{
1. Introducción
}

Que los tribunales aplican derecho es un lugar común. Decir lo que eso implica, sin embargo, no es fácil. Mi objetivo en este artículo es explicar la noción; aclarar qué es aplicar derecho.

Se presentan en simultáneo tres conjuntos de preguntas. Uno se refiere a lo que llamaré el objeto directo de la aplicación del derecho. Cuando un tribunal aplica el derecho, ¿qué es exactamente lo que aplica? No es una pregunta capciosa. Se dice que los tribunales "aplican" todo tipo de cosas: 
estatutos, reglas, principios, doctrinas, opiniones de otros tribunales, y más. ¿Es "el derecho" el nombre de otro elemento en esta lista? Eso suena erróneo. ¿O es mediante la aplicación de alguno o cualquiera de esos elementos que los tribunales aplican el derecho? Y si es así, ¿podemos dar un criterio para determinar lo que contiene la lista?

Un segundo grupo de interrogantes se refiere al objeto indirecto de la aplicación del derecho. Los tribunales no solo aplican derecho, sino que lo aplican $a$ algo. ¿A qué, exactamente? ¿A casos? ¿A hechos? ¿A los "hechos del caso particular" que tienen ante sí, como suele decirse? También decimos que un tribunal aplica el derecho en un determinado caso, y al decidir un determinado caso. ¿Es todo esto lo mismo? ¿Y qué es precisamente aplicar derecho $a$ un caso? En efecto, ¿qué es un "caso"?

En tercer lugar, preguntas sobre el contenido de la aplicación del derecho. ¿Es "aplicar el derecho" el nombre de un tipo específico de acto? Si es así, ¿cuáles son sus características distintivas? Si no es así, ¿qué es entonces? También hay cosas que se dice que los tribunales hacen - decisiones que se dice que emiten-al aplicar el derecho. ¿Son estos también actos de aplicación del derecho?

No hay un orden obvio para abordar estas cuestiones. Cualquier intento de tratar las preguntas en uno de los conjuntos se beneficiaría de haber abordado primero las de los otros. Aun así, necesitamos una estrategia, y la mía será dejar la primera serie de preguntas para el final. No discutiré explícitamente el objeto directo de la aplicación del derecho sino hasta el final del artículo; y hasta entonces, por conveniencia, me centraré en la aplicación de disposiciones legislativas y otras disposiciones jurídicas escritas, casos indiscutibles de aplicación del derecho.

No puedo, sin embargo, comenzar simplemente por cualquiera de los otros dos grupos de preguntas. Ello se debe a que hay -o así quiero sugerir- dos tipos de aplicación del derecho, cada uno dando lugar a sus propias cuestiones de contenido y objeto indirecto. Así que debo empezar por explicar cuáles son los dos tipos. Los llamo aplicación inferencial del derecho y aplicación pragmática del derecho. Analizo la primera en la sección 2, la segunda en la sección 3, y la relación entre ambas en la sección 4. En la sección 5 examino la noción de "caso"; y vuelvo, en la sección 6 , a nuestros tres conjuntos iniciales de preguntas. 


\section{Aplicación inferencial del derecho}

\subsection{Un ejemplo para la discusión}

Consideremos este extracto de la opinión de Lord Denning en The Hollandia [1982] Q.B. 872 (en 884A-B):

El artículo III, párrafo 8 [de las Reglas de La Haya-Visby para el transporte marítimo internacional de mercancías, un convenio internacional al que una ley del Reino Unido en 1971 ha dado "fuerza de ley"] ... es de primera importancia en el presente caso. Dice: "Toda cláusula ... que atenúe esa responsabilidad [es decir, la del porteador por pérdida o daño de la mercancía] de forma distinta a la prevista en estas reglas, será nula y sin efecto."

Ahora, aplíquese ese artículo III, párrafo 8 a la cláusula 2 de este conocimiento de embarque [emitido por los demandados]. En la medida en que la cláusula 2 limita la responsabilidad del porteador a D.fl. 1.250, es claramente nula y sin efecto: porque atenúa la responsabilidad del porteador a mucho menos que su responsabilidad en virtud del apartado (a) del párrafo 5 del artículo IV de las Reglas de La Haya-Visby, que asciende a 11.490,96 libras esterlinas.

$(\ldots)$

Mi conclusión es que, en los procedimientos ante los tribunales del Reino Unido, la cláusula 2 de este conocimiento de embarque es nula y sin efecto.

Es indiscutible, creo, que Denning aplicó el artículo III, párrafo 8 de las Reglas de La Haya-Visby a la cláusula 2 del conocimiento de embarque que tenía ante sí. Lo diríamos incluso si Denning no hubiera utilizado explícitamente ese lenguaje. Así que tomaré esto como ejemplo para la discusión. Nos da una clara ilustración de un sentido en el que hablamos y pensamos sobre la aplicación del derecho.

¿Qué implica entonces? 
¿Qué es aplicar derecho?

\subsection{Un acto mental}

Una cosa que no implica es la presentación de un argumento de ningún tipo. Denning, por supuesto, ofrece en aquel pasaje un argumento para una determinada conclusión. Su conclusión es que la cláusula 2 del conocimiento de embarque es nula y sin efecto. Pero este argumento no forma parte de su aplicación del artículo III, párrafo 8 de las Reglas a esa cláusula.

La aplicación de esa disposición por parte de Denning es un acto puramente mental. Es el acto de razonar hacia aquella conclusión — que la cláusula es nula y sin efecto- sobre la base de algún vínculo que él considera que existe entre la disposición y la cláusula en cuestión. Esto no significa que la creencia de Denning sobre esa conclusión deba ser el producto de una reflexión sobre la disposición pertinente. Podría haberse formado justificadamente tal creencia por motivos ajenos al artículo III, párrafo 8 de las Reglas de La Haya-Visby. Pero sí significa que Denning debe haber creído que la disposición y la cláusula estaban relacionadas de tal manera que su conclusión no podía ser falsa.

Así que cuando Denning llega a dar públicamente, por escrito, un argumento para su conclusión - cuando pasa a justificar esa conclusión a sus lectores-, ya ha aplicado la disposición. Aquello que escribe documenta su línea de razonamiento. Es evidencia, para nosotros, de cómo pensó. Pero aún habría aplicado el artículo III a la cláusula pertinente (tanto si hubiéramos podido saberlo como si no) si no hubiera presentado ningún argumento, o si se hubiera negado a hacerlo, por la razón que fuese.

Aplicar una disposición a un determinado objeto $x$-en el sentido en que diríamos de Denning que aplicó el artículo III a la cláusula del conocimiento de embarque- es algo que podemos hacer simplemente pensando. Es razonar hacia una determinada conclusión sobre $x$.

\subsection{Aplicación y aplicabilidad}

Si la aplicación de esa disposición supuso, para Denning, razonar hacia la conclusión de que la cláusula era nula y sin efecto, entonces, acabo de afirmar, debe haber considerado que estos dos elementos - disposición y 
cláusula- están vinculados de cierta manera. ¿De qué manera? ¿Qué papel juega en ese el razonamiento la disposición que se está aplicando?

La respuesta es, creo, que aplicar una disposición $p$ a un determinado objeto $x$ es razonar hacia una conclusión específica $c$ acerca de $x$ sobre la base de que (a) $p$ se aplica a $x$, y (b) si $p$ se aplica a $x$, entonces $c$ es verdadera como cuestión de derecho. Denning no utiliza explícitamente el lenguaje de la aplicabilidad, pero no habría sido sorprendente que lo hiciera. Su conclusión debe haberse basado en que el párrafo 8 del artículo III se aplica a la cláusula que tenía ante sí.

¿Es lícito que yo explique lo que es que una persona aplique una disposición $p$ a un determinado objeto $x$ diciendo que esa persona debe considerar que $p$ se aplica a $x$ ? No hay circularidad. No es lo mismo aplicar una disposición a $x$ y que una disposición se aplique a $x$. Decir que Denning aplicó el párrafo 8 del artículo III a la cláusula es decir algo sobre lo que Denning ha hecho. Decir que el párrafo 8 del artículo III se aplica a aquella cláusula es decir algo sobre cómo se relaciona la disposición con la cláusula: es decir que la disposición es (o era) aplicable a ella. Pero necesitamos, por supuesto, ser más claros sobre esta noción.

Mi posición es la siguiente. En primer lugar, decir que una disposición $p$ se aplica a un objeto $x$ es decir que hay una consecuencia jurídica específica (o consecuencias jurídicas específicas) que la existencia misma de $p$ tiene para $x$ como cuestión de derecho. Es, por lo tanto, decir que hay una afirmación específica sobre $x$ que es verdadera como cuestión de derecho, y que no podría, dada $p$, ser falsa.

Pero uno puede saber que una disposición $p$ se aplica a un objeto $x$ y no saber exactamente cuál es la consecuencia que tiene $p$ para $x$ como cuestión de derecho. Es decir, uno puede saber que existe alguna afirmación sobre $x$ que, dada $p$, debe ser verdadera como cuestión de derecho y, sin embargo, no saber exactamente cuál es esa afirmación. Y lo inverso también es posible. Uno puede saber que, si $p$ es aplicable a $x$, entonces una afirmación $c$ sobre $x$ es verdadera; pero no saber si $p$ se aplica a $c$.

Aplicar $p$ a $x$ es resolver estas dos cuestiones. Es formarse una opinión razonada sobre la consecuencia (o consecuencias) que $p$ tiene para $x$ como cuestión de derecho. 
La aplicación de Denning del párrafo 8 del artículo III de las normas de La Haya-Visby a la cláusula que tiene ante sí se traduce en el proceso de formarse su creencia de que dicha disposición tiene como consecuencia la nulidad de la cláusula.

\subsection{Enunciados de aplicabilidad}

He dicho que aplicar una disposición a un determinado objeto $x$ y llegar así a una determinada conclusión sobre $x$ no tiene por qué ser acompañado de un argumento para dicha conclusión. Pero podemos aprender algo sobre la aplicación del derecho si consideramos cómo reconstruir tal argumento cuando de hecho sea dado.

¿Cómo deberíamos pensar sobre la estructura del argumento de Denning, el argumento que da en el pasaje arriba citado? Podría ser tentador reconstruirlo de la siguiente manera:

(1) Para todo $x$, si $x$ es una cláusula de un contrato de transporte que atenúa la responsabilidad del porteador por los daños a las mercancías de forma distinta a la prevista en las Reglas de La Haya-Visby, entonces $x$ es nula y sin efecto.

(2) La cláusula 2 del conocimiento de embarque expedido por los demandados es una cláusula de un contrato de transporte que atenúa la responsabilidad del porteador por los daños a las mercancías de forma distinta a la prevista en las Reglas de La Haya-Visby.

Por lo tanto (de (1) y (2)),

(3) La cláusula 2 del conocimiento de embarque emitido por los acusados es nula y sin efecto.

Se trata de un ejemplo de lo que comúnmente se denomina un "silogismo jurídico" (o "silogismo judicial"): un argumento deductivamente válido que combina, como premisas, (a) una proposición jurídica general de primer orden (la "premisa mayor", como se la denomina normalmente - si bien de manera inexacta-) y (b) una proposición de hecho sobre algún aspecto del caso en cuestión que instancia el antecedente de aquel 
enunciado jurídico general (la "premisa menor"); y cuya conclusión es una proposición jurídica acerca de ese mismo aspecto del caso particular.

El modelo de silogismo jurídico - ampliamente adoptado por los teóricos del derecho- pretende captar la estructura de la justificación de las decisiones judiciales que, como la de Denning, aplican derecho. Este modelo va de la mano con la idea de que aplicar derecho es (para citar una formulación reciente) "resolver una disputa jurídica por medio de subsumir un caso individual bajo una premisa normativa general (una regla jurídica, una estándar normativo, un principio jurídico, un precedente, etc.)" (Moreso y Chilovi, 2018, p. 495). Sin embargo, como he argumentado en otro trabajo, el modelo del silogismo jurídico es incapaz de alcanzar sus propias metas y debe ser rechazado. ${ }^{1}$ Según la teoría que subyace al modelo del silogismo jurídico, una premisa como (1) presenta una enunciación de la "regla" expresada por la disposición que Denning estaba aplicando. Pero no hay razón para atribuir a Denning un compromiso con la verdad de algo parecido a lo que se dice en esa premisa. Denning no dice, ni tiene necesidad de decir, y con toda probabilidad no querría decir, que todas las cláusulas que atenúan responsabilidad en contratos de transporte son nulas y sin efecto: todas ellas, independientemente del contexto o de las circunstancias, sin lugar a posibles calificaciones o cláusulas adicionales de ningún tipo. Tampoco dice que la consecuencia de aplicar dicha disposición deba ser siempre, o solo, la nulidad de alguna cláusula de restricción de responsabilidad en un contrato de transporte.

La cuestión, nótese, no es que el lenguaje de (1) se apega demasiado al lenguaje de la disposición misma, mientras que un universal adecuadamente calificado tendría, muy probablemente, que alejarse de dicho texto. (Un "universal adecuadamente calificado" es uno que - si es que se puede dar, lo que en sí mismo es poco probable- podría plausiblemente verse como un enunciado jurídico verdadero). La cuestión, más bien, es que Denning simplemente no se compromete con ningún enunciado universal de ese tipo, por muy cualificado que sea.

Y lo mismo ocurre con cualquier juez. Cuando abordan la cuestión de si una disposición se aplica al caso que se tiene enfrente, los tribunales no 
formulan $-\mathrm{y}$ probablemente ni siquiera serían capaces de hacerlo- descripciones rigurosas de las propiedades que tendría que satisfacer cualquier caso al cual sea aplicable la disposición. Ni siquiera es razonable esperar que los tribunales formulen descripciones rigurosas de las propiedades relevantes del caso particular que estén tratando. No articulan ni se comprometen, como tampoco lo hace Denning, con enunciados de primer orden de las "reglas" que entienden que la disposición relevante expresa, y bajo la cual simplemente subsumirían el caso que están tratando.

Sin embargo, es esa disposición la que Denning está aplicando. Es por referencia a ella que sostiene la conclusión de que la cláusula del conocimiento de embarque es nula y sin efecto; que la disposición se aplique a la cláusula es lo que apoya su conclusión. ¿Cómo deberíamos entonces reconstruir argumentos como estos — como el de Denning-?

Se podría pensar en diferentes alternativas. ${ }^{2}$ Esto es lo que sugiero:

$\left(1^{\star}\right)$ Si el párrafo 8 del artículo III de las Reglas de La Haya-Visby se aplica a la cláusula 2 del conocimiento de embarque expedido por los demandados, entonces la cláusula 2 del conocimiento de embarque expedido por los demandados es nula y sin efecto.

$\left(2^{\star}\right)$ El párrafo 8 del artículo III de las Reglas de La Haya-Visby se aplica a la cláusula 2 del conocimiento de embarque expedido por los demandados.

Por lo tanto $\left(\operatorname{de}\left(1^{*}\right)\right.$ y $\left.\left(2^{*}\right)\right)$,

$\left(3^{\star}\right)$ La cláusula 2 del conocimiento de embarque emitido por los acusados es nula y sin efecto.

Hay dos puntos a destacar aquí. El primero es que la premisa $\left(2^{\star}\right)$ es - como también lo es, por lo tanto, el antecedente del condicional en $\left(1^{\star}\right)$ — una afirmación de segundo orden acerca de la aplicabilidad de una determi-

2 Incluyendo la posibilidad de caracterizar dichos argumentos como no deductivos. Aquí, sin embargo, asumo que la justificación de las decisiones que aplican derecho puede entenderse como una inferencia deductiva: esa es también la opinión dominante; pero en este punto creo que la visión dominante está fundamentada. (Esto no quiere decir que todos los argumentos jurídicos se caractericen mejor como deductivos). Para referencias, véase de nuevo Duarte d'Almeida, 2019. 
nada disposición a un determinado objeto $x$. Es lo que propongo llamar un enunciado de aplicabilidad. Digo que es una afirmación de segundo orden porque - a diferencia de la "premisa mayor" del silogismo jurídico, que pretende ser un enunciado de una "regla" supuestamente expresada por la disposición que se está aplicando- la premisa $\left(2^{\star}\right)$ es un enunciado acerca de la disposición misma y de cómo se relaciona con un determinado objeto.

En segundo lugar, el argumento en la reconstrucción que propongo es enteramente sobre la relación entre la disposición y un objeto particular. No es un argumento sobre las cláusulas (en plural) de conocimientos de embarque (en general); ni sobre la gama de efectos que el párrafo 8 del artículo III de las Reglas podría tener sobre cualquier cláusula de ese tipo. Se trata de un argumento sobre una cláusula determinada - la cláusula 2 del conocimiento de embarque que Denning tenía ante sí- y sobre el efecto de la disposición sobre ella. El hecho de que el párrafo 8 del artículo III de las Reglas tenga o no el mismo efecto, o un efecto diferente, o ningún efecto, sobre otras cláusulas de derecho extranjero en otros conocimientos de embarque, en circunstancias diferentes, no viene al caso, y no forma parte ni del argumento de Denning, ni de su razonamiento hacia la conclusión relevante.

Tampoco debe entenderse que la justificación del propio enunciado de aplicabilidad - la justificación de la premisa $\left(2^{*}\right)$ - implique algo análogo al silogismo jurídico; es decir, que implique la articulación de una regla general sobre la propia aplicabilidad de la disposición y cuyo antecedente esté instanciado por la cláusula del conocimiento de embarque. En otras palabras, no deberíamos tratar de reconstruir el argumento de Denning en favor de su enunciado de aplicabilidad de la siguiente manera:

(i) Para todo $x$, si $x$ es una cláusula de un contrato de transporte y $x$ restringe la responsabilidad del porteador por el daño a las mercancías de manera distinta a la prevista en las Reglas de La Haya-Visby, entonces el párrafo 8 del artículo III de las Reglas de La Haya-Visby se aplica a $x$.

(ii) La cláusula 2 del conocimiento de embarque emitido por los demandados es una cláusula de un contrato de transporte y restringe la responsabilidad del porteador por el daño a las mercancías de manera distinta a la prevista en las Reglas de La Haya-Visby. 
¿Qué es aplicar derecho?

$\left(2^{*}\right)$ El párrafo 8 del artículo III de las Reglas de La Haya-Visby se aplica a la cláusula 2 del conocimiento de embarque emitido por los demandados.

¿Cómo se justifican, entonces, en el contexto de argumentos como el de Denning, tanto los enunciados de aplicabilidad, como la premisa $\left(1^{\star}\right)$, que especifican la consecuencia que se asume que la disposición tiene para un determinado objeto si es que se aplica a él? Sobre la base de razones de diversa índole, y de cómo esas razones se relacionan con el objeto particular sobre el que se está discutiendo la aplicabilidad de la disposición. El hecho de que el texto del párrafo 8 del artículo III de las Reglas de La Haya-Visby pueda utilizarse directamente para describir la cláusula del conocimiento de embarque - para decir que esa cláusula "atenúa" la "responsabilidad del porteador" por la pérdida o el daño "de forma distinta a la prevista" en las Reglas- es, por supuesto, una de esas razones; y una razón de peso. Pero la adecuación, por muy clara que sea, al lenguaje autoritativo de una disposición, no es condición necesaria ni suficiente para justificar la afirmación de que la disposición se aplica a un determinado objeto - como demuestra, sin lugar a dudas, la práctica judicial-. Lo mismo cabe decir respecto de la premisa $\left(1^{*}\right)$. Aquí también es posible describir la consecuencia que Denning atribuye a la disposición — que la cláusula 2 es nula y sin efecto- utilizando el lenguaje que se encuentra en la disposición misma. Pero las disposiciones jurídicas pueden tener consecuencias que sus textos no mencionan e incluso pueden contradecir. Todo depende de las demás razones que sean relevantes para la cuestión, y la correspondencia con el texto de la disposición es solamente una de ellas. Basta pensar en la diversa gama de consideraciones que normalmente se denominan "cánones" de la interpretación jurídica. Estos son nada más y nada menos que tipos de razones que pueden influir en la justificación de los enunciados de aplicabilidad en argumentos como el de Denning.

La aplicación del derecho es, en cierto sentido, un asunto particularista. Lo que quiero decir no es que, para cualquier conclusión correctamente alcanzada sobre la base de que una determinada disposición $p$ se aplica a un determinado objeto $x$, no exista un principio universal verdadero que capte todos y solo aquellos aspectos de $x$ que son relevantes para esa 
conclusión - un principio que podría utilizarse para construir una inferencia que se ajustara a un esquema idéntico al del silogismo jurídico. Lo que quiero decir es que nosotros - y los tribunales en particular- no nos comprometemos (de hecho, ni siquiera podría esperarse que lo hiciéramos) con formulaciones de principios de ese tipo como un paso necesario, típico o incluso mínimamente frecuente en nuestro razonamiento o en nuestros argumentos hacia tales conclusiones.

\section{5. ¿Cualquier objeto? ¿Cualquier conclusión?}

He estado hablando de la aplicación de las disposiciones a un "determinado objeto $x$ ", y de la aplicación del derecho - en el sentido discutido hasta ahora- como razonamiento hacia una conclusión "sobre $x$ " que se considera verdadera como cuestión de derecho. Pero, ¿no hay restricciones en la gama de objetos a los que se puede aplicar una disposición? ¿Y no deberíamos decir en cambio, tal vez, que la aplicación del derecho debe ser un razonamiento hacia una proposición jurídica, o en todo caso hacia una proposición acerca del derecho "tal como aplicado" a algo?

No lo creo. "Proposición jurídica" es una frase equívoca. A veces se utiliza para referirse a las llamadas proposiciones "normativas" o "deónticas": proposiciones sobre las posiciones normativas (deberes, libertades, poderes, etc.) que tenemos en virtud del derecho vigente. Los tribunales a menudo razonan hacia proposiciones de ese tipo - "el demandado tenía el deber de prestar servicios al demandante", por ejemplo- aplicando el derecho vigente. Pero la gama de conclusiones que pueden justificarse de aquella manera no se limita a proposiciones sobre posiciones normativas. La conclusión de Denning en The Hollandia, de que la cláusula 2 de aquel conocimiento de embarque era "nula y sin efecto", no es en sí misma - al menos no obviamente - un enunciado deóntico. No hay, por lo tanto, ninguna restricción de principio sobre la gama de objetos a los que se puede (conceptualmente) aplicar el derecho existente.

Lo que sí tiene que ser el caso es que la conclusión, sea cual sea, se tome como jurídicamente verdadera, como verdadera en cuanto cuestión de derecho. Esta es una noción que no puedo analizar completamente en el presente 
trabajo. Pero un simple ejemplo sacará a relucir un punto relevante: que un enunciado puede ser presentado sea como jurídicamente verdadero, sea como verdadero tout court. Consideremos el siguiente par de disposiciones, los párrafos 1 y 2 del artículo 54 del Race Relations Act de 1976:

54. - (1) Una denuncia presentada por alguien ("el denunciante") de que otra persona ("el demandado")-

(a) ha cometido contra el denunciante un acto de discriminación que es ilegal en virtud de la Parte II; o

(b) se considera, en virtud de los artículos 32 o 33, que ha cometido tal acto de discriminación contra el denunciante,

puede ser presentada a un tribunal laboral.

(2) La sub-sección (1) no se aplica a las denuncias, presentadas bajo la sección 12(1), de un acto respecto del cual se pueda interponer una apelación, o un procedimiento en calidad de apelación, en virtud de cualquier ley, ni a las denuncias previstas en la sección 75(8).

En Khan v. General Medical Council (17 BLMR 1-10 [1993]), un médico de origen asiático apelaba contra una decisión de un tribunal. El Consejo Médico General le había denegado la inscripción como médico; y él, tras apelar sin éxito ante una Junta de Revisión, se quejó de discriminación racial indirecta ante el tribunal, con base en el artículo 54(1) del Race Relations Act de 1976. El tribunal, aplicando el artículo 54(2), desestimó la solicitud; y el Tribunal Laboral de Apelación sostuvo en Khan que aquella había sido la decisión correcta.

La conclusión justificada por la aplicación, por parte del tribunal, del artículo 54(2) a la denuncia del demandante fue que el artículo 54(1) no es aplicable a aquella denuncia. El argumento del tribunal laboral para aquella conclusión puede reconstruirse así:

(1) Si la sección 54(2) del Race Relations Act de 1976 se aplica a la denuncia del solicitante, entonces la sección 54(1) del Race Relations Act de 1976 no se aplica a la denuncia del solicitante.

(2) La sección 54(2) del Race Relations Act de 1976 se aplica a la denuncia del solicitante. 
Por lo tanto (de (1) y (2)),

(3) El artículo 54(1) del Race Relations Act de 1976 no se aplica a la denuncia del solicitante.

Aquí, tanto la premisa (2) como la conclusión son enunciados del mismo tipo. Ambos son enunciados de aplicabilidad: uno positivo, el otro negativo. Pero en el contexto de este argumento hay una diferencia crucial entre los dos. La conclusión se presenta como una afirmación que se considera jurídicamente verdadera: verdadera en virtud del derecho vigente. Se justifica, tal como la conclusión del argumento de Denning, sobre la base de que hay una determinada disposición que forma parte del derecho vigente - la sección 54(2) - que se aplica a, y tiene una determinada consecuencia para, la denuncia del demandado.

La premisa (2) también se presenta como verdadera, por supuesto; es parte del mismo argumento. Pero no es (o, en todo caso, no necesariamente) presentada como verdadera en virtud del derecho vigente. Tal vez se está presentando - como la premisa similar en el argumento de Denningsobre la base de las razones sustantivas relevantes para la aplicabilidad de la sección 54(2) a la denuncia del demandante.

Una afirmación sobre un determinado objeto $x$ se presenta como jurídicamente verdadera si se presenta sobre la base de que hay alguna parte del derecho vigente que se aplica a $x$, y cuya aplicabilidad a $x$ se considera suficiente para que aquella afirmación sea verdadera. Pero toda afirmación de aquel tipo sobre un determinado objeto $x$ se basará en última instancia en un enunciado de aplicabilidad que se presenta como verdadero, pero no verdadero como cuestión de derecho: un enunciado de aplicabilidad tal que no hay ninguna parte del derecho vigente que sea suficiente para hacerlo verdadera.

\subsection{Definición}

Nuestra discusión ha puesto de manifiesto cuatro aspectos principales de la aplicación del derecho en el sentido que hemos estado discutiendo - un sentido que propongo llamar aplicación inferencial del derecho-. Primero, 
aplicar inferencialmente el derecho es razonar de cierta manera. Segundo, es razonar hacia una conclusión sobre un determinado objeto $x$-cualquier objeto- - Tercero, es razonar hacia aquella conclusión sobre la base de que una cierta disposición $p$ se aplica, con una determinada consecuencia, a $x$. Y cuarto, se considera que la conclusión es jurídicamente verdadera, verdadera como cuestión de derecho.

He aquí, pues, una definición:

Aplicación inferencial del derecho (para disposiciones escritas): aplicar inferencialmente una disposición $p$ (por sí sola) a un determinado objeto $x$ es razonar hacia una conclusión $c$ respecto de $x$ sobre la base de que (a) $p$ se aplica a $x$, y (b) si $p$ se aplica a $x$, entonces $c$ es jurídicamente verdadera. ${ }^{3}$

Esta definición pretende captar un sentido en el que nosotros - juristas y jueces, pero también ciudadanas y ciudadanos - pensamos y hablamos comúnmente de aplicación del derecho. Pero, como mencioné, la aplicación inferencial del derecho es solo uno de dos tipos de aplicación del derecho. Consideremos ahora el otro.

\section{Aplicación pragmática del derecho}

\subsection{Definición}

Como se puede imaginar, aplicar inferencialmente el derecho no fue todo lo que Denning hizo en The Hollandia. De hecho, si Denning se hubiese limitado a razonar y argumentar hacia la conclusión de que la cláusula

3 Tanto esta definición como la de aplicación pragmática del derecho (que será ofrecida en la sección 3.1) se plantean como análisis de las respectivas nociones. La discusión hasta ahora ha buscado identificar características que nosotros, en cuanto usuarios competentes de esos dos conceptos (aunque no, claro está, bajo los nombres aquí acuñados para ellos), entendemos - y podemos, habiendo reflexionado, reconocer- que esta forma de aplicación del derecho tiene. En la siguiente sección se hará lo mismo respecto de la aplicación pragmática del derecho. Las definiciones propuestas buscan cristalizar dichas características en una formulación perspicua. 
del conocimiento de embarque era nula y sin efecto - si eso hubiese sido todo lo que hizo- habría sido acusado, con razón, de no haber aplicado el derecho al caso que tenía ante sí.

¿Qué más tenía que hacer? Tenía que admitir —y así lo hizo- la apelación:

Mi conclusión es que, en los procedimientos ante los tribunales del Reino Unido, la cláusula 2 de este conocimiento de embarque es nula y sin efecto. Esta acción judicial no debe suspenderse. Debe proceder, con el límite de responsabilidad que prescriben las Reglas de La Haya-Visby.

\section{(...)}

Admito la apelación, elimino la suspensión impuesta por el juez y permito que la acción proceda en Inglaterra (The Hollandia [1982] Q.B. 872 en $884 \mathrm{G}-885 \mathrm{~A})$.

Hay, por lo tanto, un sentido de "aplicación del derecho" que se refiere, no a un acto mental de razonamiento hacia a una conclusión, sino a la efectiva realización de una determinada acción externa, no mental, con ciertas características específicas. A esto llamaré aplicación pragmática del derecho, y propongo definir esta noción del siguiente modo:

Aplicación pragmática del derecho (para disposiciones escritas): aplicar pragmáticamente una disposición $\mathrm{p}$ es realizar una acción $\varphi$ tal que:

(a) el agente considera que $\varphi$ es una acción que o bien (a1) debe (todo considerado) jurídicamente realizar, o bien al menos (a2) le está jurídicamente permitido realizar (en el sentido de que no es el caso de que, todo considerado, no deba jurídicamente realizar);

(b) el agente considera que el juicio de deber relevante - es decir, (a1) o (a2) - se apoya en la conclusión de la aplicación inferencial de $\mathrm{p}$ a un determinado objeto $\mathrm{x} ; \mathrm{y}$

(c) realizando $\varphi$, el agente pretende estar resolviendo autoritativamente una determinada cuestión o controversia. 
¿Qué es aplicar derecho?

Como la definición deja en claro, la aplicación pragmática e inferencial del derecho están estrechamente relacionadas. Diré más sobre esta relación en la sección 4. Pero primero hay algunos aspectos de la definición que quiero discutir.

\subsection{Acción judicial y decisión judicial}

La definición menciona la realización de una determinada acción por parte de un agente. Esto podría entenderse en un sentido amplio, para incluir omisiones. Pero en el caso de los tribunales lo que está invariablemente en juego es la realización de acciones en sentido estricto, no de omisiones. Esto es porque no hacer nada es rara vez, si es que alguna vez, una verdadera opción para un juez. La opción de Denning no era admitir la apelación o simplemente no hacer nada. Era admitir o negarse a admitirla - emitiendo una decisión a tal efecto-.

"Decisión" es, en efecto, como en el discurso jurídico se suele referir a la acción pertinente. El tribunal en The Hollandia, diríamos, decidió admitir la apelación. Esto no significa normalmente mucho más que "el tribunal admitió la apelación”. Pero transmite la idea de que el tribunal estaba ocupado principalmente, como suelen estarlo los tribunales, por una pregunta normativa: ¿debería admitir la apelación? Lo que está en juego en la aplicación pragmática del derecho es la justificación jurídica de tales decisiones. Una decisión judicial $d$ está jurídicamente justificada en uno de dos escenarios: (a) Si $d$ es la decisión que el tribunal debe (todo considerado) jurídicamente emitir en el contexto decisorio relevante; o, en caso de que no haya solamente una decisión que el tribunal deba (todo considerado) jurídicamente emitir en ese contexto, (b) si $d$ no es una decisión que, todo considerado, el tribunal jurídicamente no deba emitir —es decir, si el tribunal tiene jurídicamente permitido (en un sentido de la palabra) emitirla-.

No todas las decisiones judiciales jurídicamente justificadas son decisiones que aplican derecho. Pero aquello que los tribunales deben (todo considerado) jurídicamente hacer depende en parte — en gran parte- del derecho existente. Si un tribunal, al decidir de cierta manera, aplica correc- 
tamente el derecho, eso es suficiente para que su decisión esté jurídicamente justificada.

Esto es lo que vemos en The Hollandia. Denning manifiestamente consideró que admitir la apelación era la acción que el tribunal debía (todo considerado) jurídicamente realizar; y formó esta opinión sobre la base de que había una disposición específica - el párrafo 8 del artículo III de las Reglas de La Haya-Visby - que se aplicaba a la cláusula del conocimiento de embarque. Por lo tanto, consideró que aquella decisión estaba jurídicamente justificada por referencia a aquella disposición; consideró que la disposición apoyaba normativamente - que, por así decirlo, garantizaba normativamente- la acción del tribunal de admitir la apelación.

Podemos decir, pues, que aplicar pragmáticamente una disposición es realizar una acción - emitir una decisión- que se considera jurídicamente justificada por referencia a la disposición. Pero "por referencia a la disposición” sigue siendo una descripción opaca: ese vínculo justificativo, entre disposición y acción, es lo que ahora es necesario aclarar.

\subsection{La justificación de las decisiones de aplicación de derecho}

Una forma de pensar en esta cuestión es considerar de nuevo la estructura del argumento que un juez como Denning daría para justificar su decisión: es decir, para demostrar argumentativamente que su decisión se encuentra jurídicamente justificada en cuanto decisión de aplicación del derecho. No forma parte de la definición que propuse que quien aplica pragmáticamente una disposición debe también dar un argumento que demuestre que la acción correspondiente está jurídicamente justificada. Un tribunal podría simplemente actuar de la manera descrita en la definición, y estaría aplicando derecho - incluso si fuera imposible decir, externamente, que la acción se hubiera realizado como acción de aplicación de derecho-. Pero, de nuevo, los tribunales suelen dar argumentos en ese sentido, y podemos aprender algo sobre la aplicación pragmática del derecho si reflexionamos 
sobre la estructura de esos argumentos; el vínculo justificativo entre la disposición que se aplica y la acción que se realiza se pone de manifiesto en la inferencia completamente reconstruida.

¿Cómo deberíamos entonces reconstruir el argumento de Denning? Mi sugerencia:

(1) Si el párrafo 8 del artículo III de las Reglas de La Haya-Visby se aplica a la cláusula 2 del conocimiento de embarque expedido por los demandados, entonces la cláusula 2 del conocimiento de embarque expedido por los demandados es nula y sin efecto.

(2) El párrafo 8 del artículo III de las Reglas de La Haya-Visby se aplica a la cláusula 2 del conocimiento de embarque expedido por los demandados.

Por lo tanto (de (1) y (2)),

(3) La cláusula 2 del conocimiento de embarque expedido por los denunciados es nula y sin efecto.

(4) Si la cláusula 2 del conocimiento de embarque expedido por los demandados es nula y sin efecto, y no hay consideraciones compensatorias [i.e., consideraciones de igual o mayor fuerza en sentido contrario], entonces, todo considerado, debemos [es decir, el tribunal debe] jurídicamente admitir la apelación y retirar la suspensión de la acción del demandante.

(5) No hay consideraciones compensatorias.

Por lo tanto (de (3)-(5)),

(6) Todo considerado, debemos jurídicamente admitir la apelación y eliminar la suspensión de la acción del demandante.

Se trata de un argumento deductivo complejo, en dos pasos. El primer paso - de (1) y (2) a (3) - es el argumento que corresponde, como vimos en la sección 2, a la aplicación inferencial de la disposición a la cláusula del conocimiento de embarque. La premisa (2) es el enunciado de aplicabilidad relevante; la conclusión, un enunciado sobre el efecto que se considera que la disposición tiene sobre la cláusula. Pero es el segundo paso — de (3), (4) y (5) a (6) - el que nos concierne en este momento. 
La premisa (4) es la crucial. Lo que pretende captar es que lo que se afirma en (3) - que describe el resultado de la aplicación inferencial de la disposición a la cláusula - es tomado por el tribunal como una razón para practicar una determinada acción. La premisa (4) identifica la acción - admitir la apelación y eliminar la suspensión de la acción del demandante- que cuenta, en este contexto decisorio particular, como la acción de aplicar la disposición; y señala, con la cláusula "no hay consideraciones compensatorias", que el hecho de que la cláusula en el conocimiento de embarque sea nula y sin efecto apoya normativamente - aunque no establezca de manera concluyente- la conclusión de que el tribunal debe jurídicamente admitir la apelación.

Lógicamente hablando, por supuesto, la inferencia podría comprimirse. Si un tribunal está dispuesto a adoptar las premisas (1) y (4), entonces podría afirmar directamente el siguiente condicional, que se sigue lógicamente (por silogismo hipotético) de aquellas dos afirmaciones:

$\left(1^{*}\right.$ Si el párrafo 8 del artículo III de las Reglas de La Haya-Visby se aplica a la cláusula 2 del conocimiento de embarque expedido por los demandados, y no hay consideraciones compensatorias, entonces, todo considerado, debemos jurídicamente admitir la apelación y retirar la suspensión de la acción del demandante.

Junto con (2) y (5), esta afirmación implica la conclusión final. El tribunal, por lo tanto, podría haber ofrecido simplemente la siguiente inferencia en un solo paso, en lugar de aquella más compleja que he dado anteriormente:

$\left(1^{*}\right)$ Si el párrafo 8 del artículo III de las Reglas de La Haya-Visby se aplica a la cláusula 2 del conocimiento de embarque expedido por los demandados, y no hay consideraciones compensatorias, entonces, todo considerado, debemos jurídicamente admitir la apelación y retirar la suspensión de la acción del demandante.

(2) El párrafo 8 del artículo III de las Reglas de La Haya-Visby se aplica a la cláusula 2 del conocimiento de embarque expedido por los demandados. 
¿Qué es aplicar derecho?

(5) No hay consideraciones compensatorias.

Por lo tanto (de $\left.\left(1^{\star}\right),(2), \mathrm{y}(5)\right)$,

(6) Todo considerado, debemos jurídicamente admitir la apelación y eliminar la suspensión de la acción del demandante.

Esto serviría, argumentativamente, pero solo si ni (1) ni (4) de la versión más compleja del argumento fueran afirmaciones controvertidas en el contexto decisorio. En cualquier caso, el tribunal tendría que estar preparado para desarrollar y defender el paso del enunciado de aplicabilidad - premisa (2) - al juicio de deber en la conclusión; y la forma de hacerlo sería especificando la afirmación sustantiva que es la conclusión de la aplicación inferencial de la disposición a la cláusula. ${ }^{4}$

\subsection{Apoyo}

Mencioné que, en el argumento complejo anterior, la premisa (4) presenta el hecho de que la cláusula del conocimiento de embarque es nula y sin efecto como una razón que apoya la decisión del tribunal respecto de admitir la apelación. ¿Por qué simplemente como una razón y no como un hecho suficiente para establecer que admitir la apelación era lo que, todo considerado, el tribunal debía jurídicamente hacer? ¿No estaba el tribunal

4 En las páginas 203-207 de "Applicability and Effectiveness of Legal Norms" (1997), Pablo E. Navarro y José Juan Moreso distinguen entre la aplicabilidad "interna” y la aplicabilidad "externa" de las normas jurídicas. La primera noción pretende captar lo que llamé —en la sección 2, al tratar la aplicabilidad inferencial del derecho- aplicabilidad tout court; se trata de una relación conceptual entre una norma jurídica y un caso individual (véase también Navarro y Rodríguez, 2014, pp. 126-129). La aplicabilidad “externa”, por el contrario, es una noción normativa: decir que una norma $N$ es aplicable externamente a un determinado caso genérico $C$ es, en su definición, decir que la aplicación de $N$ a los casos individuales que son instancias de $C$ es requerida (o al menos permitida) por alguna otra norma. Pero esto no es suficientemente fino: la noción de aplicabilidad externa — con su referencia genérica a la aplicación de una norma, un concepto que no exploran-pasa por alto la distinción entre la aplicación inferencial y la aplicación pragmática del derecho. Creo que Navarro y Moreso solo tienen en mente este último tipo de aplicación. 
jurídicamente obligado a permitir la apelación si la cláusula era nula y sin efecto?

No. Por una parte, la aplicabilidad del párrafo 8 del artículo III de las Reglas de La Haya-Visby, con su efecto de anular la cláusula, es compatible con la existencia de otras partes del derecho positivo - e incluso de otras disposiciones aplicables a la misma cláusula- que podrían también ser relevantes para la cuestión, y que podrían dar razones para no admitir la apelación (o al menos razones que podrían apoyar la conclusión de que no es el caso de que, todo considerado, el tribunal deba admitirla); puede haber, en el derecho, disposiciones aplicables que estén en conflicto pero igualmente válidas. Y, de forma más general, incluso en ausencia de una tal disposición conflictiva, podría ser que el tribunal tuviera jurídicamente permitido no admitir la apelación en nombre de alguna razón jurídicamente relevante de igual o mayor fuerza en sentido contrario. Lo que, todo considerado, un tribunal debe jurídicamente hacer nunca está determinado únicamente por el derecho positivo aplicable.

Por "razón" no me refiero solo a una consideración que cuente a favor o en contra de decidir en cierto modo. Consideremos una disposición como las siguiente (del Criminal Justice (Scotland) Act de 2016):

35 Autorización para el interrogatorio

(1) El tribunal puede autorizar a un agente de policía a interrogar a una persona sobre un delito después de que la persona haya sido oficialmente acusada de cometer el delito...

Esta disposición, que confiere una libertad (es decir, una permisión), es una disposición que un tribunal que decida autorizar dicho interrogatorio puede aplicar pragmáticamente. El tribunal podrá correctamente considerar no solo que su decisión está, todo considerado, jurídicamente permitida (y, por lo tanto, que no es el caso que, todo considerado, el tribunal jurídicamente no deba emitirla), sino también que la disposición apoya (aunque, de nuevo, no de manera concluyente) esa proposición acerca de lo que le está, todo considerado, jurídicamente permitido hacer. Pero no se sigue, y no es necesariamente verdadero, que, todo considerado, el tribunal deba jurídicamente autorizar el interrogatorio. Puede no ser verdadero ni que, 
todo considerado, deba, ni que no deba hacerlo (lo que bastaría para que la decisión estuviese jurídicamente justificada); y de todas maneras aquella disposición no proporciona al tribunal ninguna razón para decidir en cualquiera de los sentidos. No todos los hechos que apoyan proposiciones acerca de qué, todo considerado, debe (o no) hacerse son razones a favor o en contra de algo; y por "razón" me refiero a un hecho que apoya una proposición acerca de qué, todo considerado, debe (o no) hacerse. ${ }^{5}$

Nótese, de paso, que la conclusión de la aplicación inferencial de una determinada disposición bien puede ser directamente un enunciado acerca de la acción misma que el tribunal debe jurídicamente realizar si quiere aplicar pragmáticamente la disposición. Ello es lo que observamos en el ejemplo que se acaba de presentar. La disposición que justifica (a falta de consideraciones de igual o mayor fuerza en sentido contrario) la decisión del tribunal de autorizar a un agente de policía a interrogar a alguien es ella misma una disposición sobre las decisiones de los tribunales de autorizar a los agentes de policía a interrogar a alguien. (En The Hollandia, en cambio, la disposición que se aplica se refiere a las cláusulas de limitación de la responsabilidad en los contratos de transporte, y no a si se deben denegar determinadas apelaciones). Pero aun en esos casos debemos diferenciar entre la conclusión de la aplicación inferencial de la disposición en una determinada situación particular ("este tribunal tiene la libertad de autorizar a un agente de policía a interrogar a esta persona”, por ejemplo), y el juicio de deber que aquella conclusión apoya ("no es el caso de que este tribunal, todo considerado, legalmente no deba autorizar a un agente de policía a interrogar a esta persona"). El enunciado condicional "si la sección 35(1) del Criminal Justice (Scotland) Act de 2016 se aplica a la presente

5 Junto a Euan MacDonald proponemos una caracterización de la noción de razón en términos de la relación de "apoyo" ("support") — que se centra en la relación entre los hechos que constituyen razones y el valor de verdad de enunciados de deber todo considerado ["all things considered"] (en contraposición a la relación de "favorecimiento" ["favouring"], que se centra en la relación entre los hechos que constituyen razones y aquello para lo que son razones - acciones, creencias, etc.) - en nuestro artículo "Contra Tantum Reasons" (inédito, y todavía en progreso). A los efectos presentes, sin embargo, basta decir que mi uso de "razón" acá abarca tanto favorecedores (incluyendo razones tanto a favor como en contra de cierto curso de acción) como apoyantes ["supporters"]. 
situación, entonces no es el caso que este tribunal, todo considerado, no deba autorizar a un agente de policía a interrogar a esta persona" no es una tautología.

\subsection{Actuar en calidad de autoridad}

Sin embargo, lo que hace que la acción de Denning — su decisión — sea un acto de aplicación del derecho no es simplemente el hecho de que lo haya entendido como una acción que, todo considerado, debía jurídicamente realizar. No sería un acto de aplicación del derecho si no se hubiera realizado - o, al menos, si Denning no hubiera pretendido realizarlo- en el cumplimiento de una función específica (la de un juez, en su caso) y como medio de poner fin de manera autoritativa (aunque de forma solo provisional) a un determinado asunto o controversia.

Algunos autores señalan que, como ciudadanas y ciudadanos, podemos relacionarnos con el derecho de muchas maneras - podemos seguirlo, cumplirlo, romperlo, invocarlo, etc. - pero no exactamente "aplicarlo". ${ }^{6}$ Esto es cierto solo en parte, como sabemos ahora: a menudo, como meras y meros ciudadanas y ciudadanos, sí aplicamos inferencialmente el derecho. Suponga que quiere casarse, pero, habiendo obtenido recientemente un divorcio, se pregunta si es jurídicamente posible casarse de nuevo tan pronto. Así que lee la legislación pertinente, y concluye que sí se le permite volver a casarse. Usted ha aplicado — aplicado inferencialmente- la disposición o disposiciones pertinentes para llegar a aquella conclusión. Pero es cierto que al seguir adelante y casarse, no estará realizando otro acto de aplicación del derecho; simplemente ejercerá un poder jurídico y se aprovechará de la libertad jurídica que lo acompaña. ${ }^{7}$ De igual manera, puedo, aplicando inferencialmente una determinada disposición, llegar a la conclusión de que jurídicamente tengo el deber de pagarle una cierta

6 Véase, por ejemplo, MacCallum, 1993, pp. 63, 72-4; Pino, 2011, pp. 802-809; Carpentier, 2018, pp. 109-111. 
cantidad de dinero; pero al pagarla no estoy "aplicando" la ley: simplemente estoy cumpliendo con mi deber.

No es en nuestra calidad de ciudadanas y ciudadanos que aplicamos pragmáticamente disposiciones jurídicas. ${ }^{8}$ Solo aplicamos pragmáticamente una disposición jurídica cuando, en alguna función o calidad específica - como es, paradigmáticamente, la de un juez- ejercemos (o al menos pretendemos ejercer) la autoridad para llevar una determinada cuestión o controversia a una resolución de algún tipo. La acción pertinente -la decisión - tiene por objeto resolver esa cuestión, y el agente la considera justificada por referencia a la disposición.

\section{4. ¿Cómo se relacionan la aplicación inferencial y la aplicación pragmática del derecho?}

La aplicación pragmática del derecho presupone conceptualmente - como mi definición lo aclara- la aplicación inferencial. Pero hay más que decir sobre las relaciones entre los dos tipos de aplicación.

En primer lugar, quien aplica pragmáticamente una disposición $p$ a un determinado objeto $x$ no tiene por qué ser la misma entidad que la ha aplicado inferencialmente. Típicamente, por supuesto, será el mismo juez o tribunal el que aplicará inferencialmente una disposición a un determinado objeto $x$, arribando a la conclusión correspondiente, y luego pasará a aplicar pragmáticamente aquella disposición, realizando la acción que esa conclusión normativamente apoya. Pero las dos operaciones pueden recaer en entidades diferentes. Ello es lo que parece ser el caso, por ejemplo,

8 Esto no quiere decir que nunca llevemos a cabo, como ciudadanas y ciudadanos, acciones que podrían llamarse acciones de aplicación del derecho en algún sentido de la noción - un sentido no reducible a nociones tales como cumplir con la ley, seguir al derecho, hacer lo que el derecho requiere, etc.-. Consideraciones como las que acabo de mencionar no son suficientes para resolver este punto. Pero no es un punto que pretenda resolver en este artículo. Acá estoy enfocado en el sentido particular y distintivo en el que decimos de los tribunales (y otros agentes institucionales) que característicamente aplican derecho. Pensamos en los tribunales como órganos que aplican derecho; y lo que ello significa no es que simplemente pase que los tribunales apliquen — como podría hacerlo cualquier otra persona o entidad- el derecho. 
cuando un tribunal nacional de un Estado miembro de la Unión Europea remite al Tribunal de Justicia de la Unión Europea una cuestión interpretativa del derecho de la Unión Europea. El fallo del Tribunal de Justicia puede conllevar la aplicación inferencial del derecho de la Unión Europea; y el tribunal nacional se basará entonces en ella para proceder a la aplicación pragmática del derecho pertinente. ${ }^{9}$

En segundo lugar, la aplicación pragmática y la aplicación inferencial del derecho no son reducibles, desde el punto de vista de su explicación, la una a la otra. ¿Por qué alguien podría pensar que, dadas mis definiciones, la aplicación pragmática del derecho es reducible a la aplicación inferencial? La idea sería que la aplicación pragmática de una determinada disposición $p$ podría entenderse en términos de aplicación inferencial de otra cosa: de la regla putativamente jurídica de que, si $p$ se aplica a un determinado objeto, entonces, en ausencia de consideraciones de igual o mayor fuerza en sentido contrario, el tribunal debería decidir jurídicamente (o al menos que no es el caso que no debería decidir jurídicamente) de una determinada manera. La aplicación pragmática de $p$ sería entonces explicable como (a) la aplicación inferencial de aquella supuesta regla, simplemente unida a (b) la realización efectiva de la acción pertinente por parte del tribunal. En The Hollandia, por ejemplo, la aplicación pragmática del párrafo 8 del artículo III de las Reglas de La Haya-Visby por parte del tribunal podría explicarse - así se pensaría - como la aplicación inferencial de la regla de que, si esa disposición se aplica a la cláusula del conocimiento de embarque, entonces, si no hay consideraciones de igual o mayor fuerza en sentido contrario, el tribunal debería jurídicamente permitir la apelación. Habiendo razonado para llegar a esta conclusión — que parece ajustarse a mi definición de aplicación inferencial del derecho- el tribunal simplemente actuó en conformidad. No queda nada por explicar.

Pero esta línea de razonamiento es errónea. Es cierto que al aplicar pragmáticamente una disposición $p$, un tribunal habrá razonado que, si $p$ se aplica a un determinado objeto, entonces, en ausencia de consideraciones de igual o mayor fuerza en sentido contrario, su decisión es una que jurídicamente debe emitir (o no es una que jurídicamente no deba emitir). 
Pero enunciados condicionales como estos, si son verdaderos, no son en sí mismos jurídicamente verdaderos, verdaderos en virtud del derecho. Si enuncian una regla, no es una regla que forme parte del derecho existente. Más bien, sería una regla sobre el derecho existente y sobre la forma en que el derecho existente incide en lo que el tribunal debe jurídicamente hacer; sería una regla sobre lo que se debe hacer si una cierta parte del derecho existente - la disposición $p$ - se aplica a un objeto determinado.

Para decirlo en términos más generales: la regla (o principio, si es eso lo que es) de que los tribunales deben aplicar el derecho no forma parte del derecho cuya aplicación prescribe. Esto no quiere decir que no pueda haber una regla jurídica - parte del derecho vigente- que prescriba a los tribunales aplicar el derecho existente; una regla autorreferencial incluso. Pero todavía necesitaríamos saber si los tribunales deben jurídicamente aplicar el derecho vigente, incluyendo esa regla; y si la respuesta es que sí lo deben hacer, la regla o principio que así lo determinara no sería en sí misma parte del derecho vigente. Así que la aplicación pragmática del derecho no puede explicarse en términos de aplicación inferencial del derecho. (Sin mencionar, por supuesto, que la aplicación inferencial del derecho se basa, como argumenté en la sección 2.4, en enunciados de aplicabilidad de segundo orden, y no en afirmaciones de primer orden que enuncien el contenido de lo que sea que se está aplicando).

Tampoco es posible reducir la aplicación inferencial a la aplicación pragmática del derecho. Aquí la idea sería que si la aplicación inferencial del derecho se caracteriza, como he sostenido, como realización de un acto mental, entonces podría incluirse en mi definición de aplicación pragmática, dada una lectura suficientemente amplia de "acción". Pero, de nuevo, esto no serviría. La aplicación pragmática del derecho no se caracteriza simplemente como realización de una acción, sino de una acción que el agente considera jurídicamente justificada sobre la base del derecho existente; y eso nunca es verdad respecto del acto mental de arribar a una determinada conclusión sobre un determinado objeto sobre la base del derecho existente. Tal vez sea cierto que, en The Hollandia, Denning debería haber llegado, como de hecho lo hizo, a la conclusión de que la cláusula del conocimiento de embarque era jurídicamente nula y sin efecto. Pero el 
acto mental de llegar a aquella conclusión no es en sí mismo un acto que se justifique por referencia al derecho existente. Más bien se justifica por referencia a las consideraciones sustantivas (y lógicas) que rigen la forma en que los tribunales deben basarse en el derecho existente para razonar hacia conclusiones sobre objetos como las cláusulas de los conocimientos de embarque.

Sin embargo, los dos tipos de aplicación del derecho están relacionados no solo conceptualmente sino también normativamente. En la sección 2.4 mencioné que la justificación de los enunciados de aplicabilidad (y de las afirmaciones sobre sus consecuencias) se basa en una serie de consideraciones sustantivas, a las cuales un tribunal debe ser sensible. Entre ellas figuran las consecuencias adicionales que se derivarán (o derivarían) si la conclusión que resulta de la aplicación inferencial de esa disposición realmente se considera verdadera en términos jurídicos, y si se actúa en consecuencia. En otras palabras, el hecho de que haya una determinada decisión $d$ que el tribunal deba jurídicamente emitir si una disposición $p$ se aplica con una determinada consecuencia a un objeto $x$ es en sí mismo una consideración que debe tener en cuenta el tribunal al determinar si $p$ se aplica o no a $x$. Si $d$ es una decisión sumamente indeseable o absurda, entonces esa es una consideración que en sí misma incide en el razonamiento del tribunal sobre si se aplica o no la disposición pertinente. La aplicación pragmática del derecho, para decirlo de modo sintético, retroalimenta normativamente la aplicación inferencial del derecho.

\section{Casos}

Como mencioné en la sección 1, se suele decir que los tribunales aplican el derecho tanto a como en casos. Hasta ahora he intentado evitar el lenguaje de "casos", y lo he logrado casi siempre. Pero las definiciones que propongo deberían al menos ser compatibles con ese lenguaje. ¿Lo son?

La noción de caso es menos clara de lo que parece. En el discurso jurídico encontramos, creo, al menos cuatro sentidos de la palabra.

A veces - llamémosle "Sentido 1"- lo que entendemos por un caso es, bueno, nada en absoluto. Consideremos la siguiente afirmación: 
¿Qué es aplicar derecho?

(A) La disposición del párrafo 8 del artículo III de las Reglas de La Haya-Visby se aplica al caso de una cláusula de derecho extranjero en un conocimiento de embarque.

Esto es algo que el tribunal de The Hollandia podría haber dicho. Pero, ¿qué es este "caso de una cláusula de derecho extranjero"? No es lo que el tribunal llamaría "el caso en cuestión"; eso no tendría sentido. La afirmación es simplemente, me parece, una perífrasis innecesaria. ${ }^{10}$ Es una forma indirecta de decir que

(B) La disposición del párrafo 8 del artículo III de las Reglas de La Haya-Visby se aplica a las cláusulas de derecho extranjero en los conocimientos de embarque,

lo que a su vez debe entenderse como la afirmación de que el hecho de que una cláusula sea una cláusula de derecho extranjero en un conocimiento de embarque no significa que la disposición no se aplique a ella.

De la misma manera,

(C) La disposición del párrafo 8 del artículo III de las Reglas de La Haya-Visby se aplica al caso de la cláusula 2 del conocimiento de embarque expedido por los demandados no significa nada más que

(D) La disposición del párrafo 8 del artículo III de las Reglas de La Haya-Visby se aplica a la cláusula 2 del conocimiento de embarque expedido por los demandados.

${ }^{10}$ Arthur Quiller-Couch hace algunos comentarios divertidos sobre la palabra "caso" - "el hijo más querido de la jerga", le llama en On the Art of Writing (1923, p. 77)-. Fowler tampoco era un fan: "quizás no hay otra palabra a la que se recurra tan libremente para ahorrar problemas, y responsable, por consiguiente, de tanta escritura flácida” (véase Fowler, 1968, pp. 76-77, y Fowler y Fowler, 1931, pp. 15-16). En caso de que se lo pregunte, Fowler no se opone a "fan". (Tampoco ha objetado — en caso de que también se lo pregunte - "en caso de que se lo pregunte": es uno de los "usos legítimos" de la palabra. "En el caso de" es, sin embargo, "el peor infractor".) 
En otro sentido de la palabra -el que está en juego cuando hablamos de un juez que decide el caso que tiene ante sí-, un caso es en realidad una pregunta particular. Es una pregunta que se le plantea a un juez y sobre la cual se espera que se pronuncie. Es siempre una pregunta normativa, y siempre se refiere a una determinada acción del juez o del tribunal. ¿Debe el juez hacer cumplir este contrato? ¿Condenar a este acusado? ¿Admitir esta apelación? "Decidir el caso" es, para el tribunal, adoptar un curso de acción particular: el curso de acción que corresponde a lo que el juez piensa que, todo considerado, debe jurídicamente hacer cuando se enfrenta a tal pregunta.

Aunque un caso en este sentido de la palabra -el Sentido 2- es una pregunta y no un conjunto de hechos, es típicamente una pregunta planteada en relación con un conjunto particular de hechos (o descripciones de hechos) que también se presentan ante un juez. Estos son lo que normalmente llamaríamos los "hechos del caso"; y tales hechos - que no son el "caso" en el sentido que acabamos de especificar- son lo que a veces también llamamos "el caso" ante el juez. Así, en este sentido -el Sentido 3 del término-, un "caso" es una serie de descripciones de hechos particulares, en relación con los cuales se ha planteado una determinada pregunta normativa que se ha llevado ante un juez para ser decidida.

En un cuarto sentido, el término se refiere más ampliamente a cualquier contexto decisorio en el que se pide a un tribunal que se pronuncie sobre un caso en el segundo sentido del término. "En este caso", el tribunal podría haber dicho en The Hollandia, refiriéndose al contexto decisorio particular en el que se encontraba, "decidimos admitir la apelación".

(Hay un quinto sentido, aunque no sea de interés para nuestros propósitos actuales; es el sentido en el que hablamos, por ejemplo, de The Hollandia en sí mismo como un "caso": un "caso" al que podemos referirnos, citar, analizar, criticar, etc. Aquí el término se refiere de manera exhaustiva a un determinado evento de toma de decisión con todos sus componentes, incluyendo la decisión emitida por el tribunal, junto con su registro en la sentencia del tribunal).

$¿$ Aplican los tribunales, entonces, el derecho $a$ o en -o al decidircasos en cualquiera de estos sentidos? Sí. En el Sentido 1, el término se utiliza para referir - aunque de manera redundante - al objeto indirecto de la aplicación inferencial del derecho: a aquello a lo que se aplica inferen- 
cialmente el derecho. En el Sentido 4, el término se refiere al contexto decisorio en el que el tribunal se enfrenta a una pregunta normativa a la que el juicio de deber de la aplicación pragmática del derecho proporciona una respuesta. Y en el sentido 2, el término se refiere a esta misma pregunta, a la cuestión en la decisión de la cual el tribunal aplica pragmáticamente el derecho.

\section{Conclusión}

Comencé este trabajo identificando tres conjuntos de preguntas sobre la aplicación del derecho: preguntas sobre lo que llamé (a) su objeto directo, (b) su objeto indirecto, y (c) su contenido. Dejé las tres para el final, aunque por diferentes razones.

He pospuesto el tratamiento de las preguntas sobre objeto indirecto y contenido porque, según expliqué, estas podrían recibir respuestas diferentes según el tipo de aplicación del derecho que tengamos en mente. Lo que acabo de decir en la sección 5 sobre la noción de caso demuestra esto con relación al objeto indirecto de aplicación del derecho. El objeto indirecto de la aplicación inferencial del derecho es un "caso" solo en el primer sentido de la palabra. Es, como vimos en la sección 2.5, cualquier objeto $x$ tal que la aplicabilidad a $x$ de una determinada parte del derecho existente es relevante para la cuestión de si cierta afirmación sobre $x$ es jurídicamente verdadera. Es a eso que un tribunal aplicará inferencialmente el derecho. En cambio, la aplicación pragmática del derecho es la aplicación no $a$ un caso sino en un caso, o en la decisión de un caso - pero en otros sentidos de "caso"- - En cuanto al contenido de cada uno de los dos tipos de aplicación del derecho, esto es precisamente lo que las definiciones que he propuesto pretenden caracterizar.

Las preguntas sobre el objeto directo de la aplicación del derecho, sin embargo, las he pospuesto solo por razones de conveniencia. El hecho de centrarme en la aplicación de disposiciones escritas me permitió utilizar ejemplos claros y sencillos mientras desarrollaba y defendía mis análisis. Pero, ¿qué podemos decir de manera más general sobre el objeto directo de la aplicación del derecho? 
Podemos decir que abarca cualquier parte del derecho existente que sea saliente en el contexto decisorio relevante: cualquier sección del derecho positivo (o incluso no positivo, según lo que permita su metafísica jurídica) que exista en el momento de la aplicación, y que pueda identificarse como tal - como parte del derecho existente- independientemente de cualquier opinión sobre su aplicabilidad y aplicación a cualquier objeto (e independientemente también de cualquier opinión sobre la conveniencia normativa de aplicarla pragmáticamente en cualquier contexto particular de adopción de decisiones). Puede tratarse de una disposición aislada, pero también, por ejemplo, de un grupo de disposiciones tomadas en conjunto, o de una decisión judicial (en sistemas jurídicos que reconozcan el principio del stare decisis), o de una costumbre -o lo que sea que cuente como parte del derecho según la teoría correcta de lo que es el derecho-.

\section{Bibliografía}

Carpentier, M. (2018). Validity versus Applicability: A (Small) Dose of Scepticism. Diritto e Questione Pubbliche, 18, 107-132.

Duarte d'Almeida, L. (2019). On the Legal Syllogism. En D. Plunkett, S. Shapiro y K. Toh (eds), Dimensions of Normativity: New Essays on Metaethics and Jurisprudence (pp. 335-364). Nueva York: Oxford University Press.

Fowler, H. W. (1968). Fowler's Modern English Usage. 2 ed. realizada por Sir Ernest Gowers. Oxford: Oxford University Press.

Fowler, H. W. y Fowler, E.G. (1931). The King's English. 3 ed. Oxford: $₫$ Oxford University Press.

MacCallum, G. (1993). On Applying Rules. En M. G. Singer y R. Martin (eds.), Legislative Intent and Other Essays on Law, Politics, and Morality (pp. 64-74). Madison: The University of Wisconsin Press.

Moreso, J. J. y Chilovi, S. (2018). Interpretive Arguments and the Application of the Law. En G. Bongiovanni et al (eds.), Handbook of Legal Reasoning and Argumentation (pp. 495-517). Dordrecht: Springer. Navarro, P. y Moreso, J. J. (1997). Applicability and Effectiveness of Legal Norms. Law and Philosophy, 16, 201-219. 
Navarro, P. y Rodríguez, J. L. (2014). Deontic Logic and Legal Systems, Cambridge: Cambridge University Press.

Pino, G. (2011). Lapplicabilità delle norme giuridiche. Diritto e Questioni Pubbliche, 11, 797-871.

Quiller-Couch, A. (1923). On the Art of Writing. Cambridge: Cambridge University Press. 